Homology, Homotopy and Applications, vol.20(2), 2018, pp.207-216

\title{
COMMUTATIVE NEARLY ABSOLUTE VALUED ALGEBRAS WITH SQUARE ROOTS
}

\section{JOSÉ ANTONIO CUENCA MIRA AND FRANCISCA MIGUEL GARCÍA}

\author{
(communicated by Winfried Bruns)
}

\begin{abstract}
Let $A$ be a commutative nearly absolute valued algebra. In this note we prove that all elements of $A$ have square roots if and only if $A$ is a two-dimensional real division algebra. This can be viewed as a generalization of a well-known theorem of Hopf.
\end{abstract}

\section{Introduction}

This note deals with commutative nearly absolute valued algebras whose elements have square roots. Let $A$ be a real or complex algebra, not necessarily associative, as are all the algebras considered throughout this paper. We recall that a real or complex algebra $A \neq 0$ is said to be a nearly absolute valued algebra if its underlying vector space is a normed vector space and, in addition, there exists a real number $k>0$ such that

$$
k\|x\|\|y\| \leqslant\|x y\| \leqslant\|x\|\|y\|
$$

for any $x, y \in A$. In the case where only the last inequality of (1) holds, $A$ is said to be a normed algebra.

Nearly absolute valued algebras have been introduced in [14] and a quick review is contained in [4, pp. 281-283]. They include finite dimensional real division algebras, because in this case the spheres are compact. Nevertheless, there exist infinitedimensional nearly absolute valued algebras which are not division algebras (see [14] or $[4])$.

We recall that if $K$ is an arbitrary field and $A$ is a $K$-algebra then for each $x \in A$ we denote by $L_{x}$ (resp. $R_{x}$ ) the linear map $L_{x}: A \rightarrow A$ (resp. $R_{x}: A \rightarrow A$ ) defined by $L_{x}: y \mapsto x y$ (resp. $\left.R_{x}: y \mapsto y x\right)$ for all $y \in A$. The linear map $L_{x}$ (resp. $R_{x}$ ) is named the left (resp. right) multiplication operator of $x$. We say that $A \neq 0$ is a division algebra if the linear operators $L_{x}$ and $R_{x}$ are bijective maps for each nonzero element $x$ of $A$.

Recalling several results from [5], we can establish the following proposition.

Proposition 1.1 ([5, Lemma 2.1 and Propositions 1.2 and 1.1]). If $A$ is a commutative nearly absolute valued division algebra then either it is isomorphic to $\mathbb{R}$ or all elements of $A$ have square roots.

Received September 27, 2017, revised February 12, 2018; published on June 6, 2018.

2010 Mathematics Subject Classification: 17A35, 55N10, 46H70.

Key words and phrases: division algebra, singular homology group, commutative algebra, square root, fundamental group.

Article available at http://dx.doi.org/10.4310/HHA.2018.v20.n2.a10

Copyright (C) 2018, International Press. Permission to copy for private use granted. 
From this a natural question arises: if $A$ is a commutative nearly absolute valued algebra whose elements have square roots, is $A$ a real division algebra of dimension 2 ? In this note we give an affirmative answer to this question (Theorem 4.1). More concretely, if $A$ is a commutative nearly absolute valued algebra, we prove that all elements of $A$ have square roots if and only if $A$ is a two-dimensional real division algebra. This can be viewed as a generalization of a celebrated theorem of Hopf asserting that every commutative finite dimensional real division algebra, $A$, has dimension $\leqslant 2$, [12]. Indeed, since $A$ is a finite-dimensional real division algebra, $A$ is a nearly absolute valued algebra. Assume $\operatorname{dim} A>1$. As a consequence of Proposition 1.1, we get the well known property that all elements of $A$ have square roots (see also $[\mathbf{1 7}$, Lemma 8.64] or [8, pp. 232, 235-236]). So our main theorem (Theorem 4.1) gives $\operatorname{dim} A \leqslant 2$.

In contrast to $[\mathbf{8}]$ or $[\mathbf{1 7}]$, where homotopical methods prevail, here the proof of our main result depends heavily on the consideration of some homology groups. So section 2 is devoted to the computation of different homology groups of topological spaces obtained from a given real normed vector space. In section 3 we show the closed character of some maps related to the square map $q: A \backslash\{0\} \rightarrow A \backslash\{0\},\left(q: x \mapsto x^{2}\right)$. Finally, in section 4 we prove our main theorem. In addition several consequences are also obtained. So using the characterization of the commutative nearly absolute valued division algebras given in [5, Theorem 2.4] it is possible to assert that for each commutative real algebra $A$ the following assertions are equivalent: (i) $A$ is a nearly absolute valued division algebra; (ii) either $A \cong \mathbb{R}$ or $A$ is a nearly absolute valued algebra with all elements having square roots; (iii) $A$ is a division algebra of dimension $\leqslant 2$.

We must point out that our main theorem allows us to determine up to isomorphism the commutative real nearly absolute valued algebras all of whose elements have square roots, because actually a complete classification of the commutative twodimensional real division algebras is well-known (see $[\mathbf{3}, \mathbf{1}, \mathbf{1 5}, \mathbf{1 1}, \mathbf{6}, \mathbf{7}]$; especially $[6]$ and $[7]$ where interesting reviews of the previous works on the topic are given). Related to this classification is Proposition 3.1 of [5], which jointly with Theorem 4.1 has as a consequence that if $A$ is a commutative nearly absolute valued algebra with all elements having square roots and satisfying $\left\|x^{2}\right\|=\|x\|^{2}$ for each $x \in A$, then $A$ is isomorphic to $\mathbb{C}$ or $\stackrel{\star}{\mathbb{C}}$ (Corollary 4.7 ).

\section{Computing some first singular homology groups}

Let $A$ be a commutative real nearly absolute valued algebra with all elements having square roots. The homological properties of some topological spaces associated with $A$ play a fundamental role in our determination of the possible values of $\operatorname{dim} A$. These topological spaces can be also defined taking as a point of departure an arbitrary real normed vector space $V$. So, given $V$, we can define the quotient topological space $Z(V)$ obtained from $V \backslash\{0\}$ by identifying each pair of opposite nonzero elements of $V$. We will use in the sequel $Z$ instead of $Z(V)$, because the reference to $V$ is here understood. The topological space $Z$ can also be seen as the space of orbits of $V \backslash\{0\}$ under the continuous action of the group of isometric transformations $H=\{I d, \tau\}$, where $\tau: V \backslash\{0\} \rightarrow V \backslash\{0\}$ is given by $\tau: x \mapsto-x$. Unfortunately the methods of algebraic topology have been applied almost exclusively to the topological 
spaces related to euclidean subspaces. Despite this fact, they can be also applied in different contexts. For the convenience to the reader we recall some of these methods, showing the way in which they can be used in the determination of the first homology groups of some topological spaces related to a given real normed vector space.

Lemma 2.1. Let $V$ be a real normed vector space with $\operatorname{dim} V \geqslant 2, H$ the group of isometric transformations of $V \backslash\{0\}$ generated by the map $\tau: V \backslash\{0\} \rightarrow V \backslash\{0\}$ given by $\tau: x \mapsto-x(x \in V \backslash\{0\})$, and $Z=(V \backslash\{0\}) / H$ its space of orbits. Then for the first integer singular homology groups $H_{1}(V \backslash\{0\})$ and $H_{1}(Z)$ we have

$$
H_{1}(V \backslash\{0\}) \cong \begin{cases}\mathbb{Z} & \text { if } \operatorname{dim} V=2 \\ 0 & \text { if } \operatorname{dim} V>2\end{cases}
$$

and

$$
H_{1}(Z) \cong \begin{cases}\mathbb{Z} & \text { if } \operatorname{dim} V=2 \\ \mathbb{Z} / 2 \mathbb{Z} & \text { if } \operatorname{dim} V>2 .\end{cases}
$$

Proof. Let $a$ (resp. $b$ ) be an arbitrary point of $V \backslash\{0\}$ (resp. $Z$ ). By the Hurewicz Isomorphism Theorem, the arcwise connected character of $S$ and $Z$ imply that $H_{1}(V \backslash$ $\{0\})$ and $H_{1}(Z)$ are isomorphic to the abelianized groups $\pi_{1}(V \backslash\{0\}, a) /\left[\pi_{1}(V \backslash\right.$ $\left.\{0\}, a), \pi_{1}(V \backslash\{0\}, a)\right]$ and $\pi_{1}(Z, b) /\left[\pi_{1}(Z, b), \pi_{1}(Z, b)\right]$. Since

$$
\pi_{1}(V \backslash\{0\}, a) \cong\left\{\begin{array}{lll}
\mathbb{Z} & \text { if } \operatorname{dim} V=2 \\
0 & \text { if } & \operatorname{dim} V>2
\end{array}\right.
$$

(see [8, p. 234]), we obtain (2). Since $Z$ is the quotient topological space obtained from $V \backslash\{0\}$ by identifying each pair of opposite points, if $p_{Z}: V \backslash\{0\} \rightarrow Z$ is the projection map then it is easy to show that $p_{z}$ is a covering map of two sheets (see [18, Proposition 4.20 and Remark 4.21(iii) below]). Now let $b=p_{Z}(a)$ and assume that $\operatorname{dim} V>2$. In this case $\pi_{1}(V \backslash\{0\}, a)=0$ and, in consequence, $\pi_{1}(Z, b)$ has two elements (see for instance [10, Proposition 1.32]). Therefore, if $\operatorname{dim} V>2$ then we have $\pi_{1}(Z, b) \cong \mathbb{Z} / 2 \mathbb{Z}$, consequently $H_{1}(Z) \cong \mathbb{Z} / 2 \mathbb{Z}$. Let us now assume $\operatorname{dim} V=2$. In this case it is easily checked that the quotient topological space $Z$ is homeomorhic with a circular cone perforated by its vertex, this last topological space being homotopy equivalent to the unit circle. Therefore, $H_{1}(Z) \cong \mathbb{Z}$ if $\operatorname{dim} V=2$.

Remark 2.2. We can point out that if $V$ is a real normed vector space of dimension $\geqslant 2$ and $Z$ is the space of orbits given as in Lemma 2.1, then the singular homology groups $H_{1}(V \backslash\{0\} ; G)$ and $H_{1}(Z ; G)$ of $V \backslash\{0\}$ and $Z$ with coefficients in the abelian group $G$ are

$$
H_{1}(V \backslash\{0\} ; G) \cong \begin{cases}G & \text { if } \operatorname{dim} V=2 \\ 0 & \text { if } \operatorname{dim} V>2\end{cases}
$$

and

$$
H_{1}(Z ; G) \cong \begin{cases}G & \text { if } \operatorname{dim} V=2 \\ G / 2 G & \text { if } \operatorname{dim} V>2\end{cases}
$$

Indeed, as a consequence of the Universal Coefficient Theorem we have

$$
\begin{aligned}
H_{1}(V \backslash\{0\} ; G) & \cong\left(H_{1}(V \backslash\{0\}) \otimes G\right) \oplus \operatorname{Tor}_{1}\left(H_{0}(V \backslash\{0\}), G\right), \\
H_{1}(Z ; G) & \cong\left(H_{1}(Z) \otimes G\right) \oplus \operatorname{Tor}_{1}\left(H_{0}(Z), G\right) .
\end{aligned}
$$


By Lemma 2.1 we now obtain

$$
H_{1}(V \backslash\{0\} ; G) \cong \begin{cases}(\mathbb{Z} \otimes G) \oplus \operatorname{Tor}_{1}(\mathbb{Z}, G) \cong G & \text { if } \operatorname{dim} V=2 \\ 0 \oplus \operatorname{Tor}_{1}(\mathbb{Z}, G)=0 & \text { if } \operatorname{dim} V>2\end{cases}
$$

and

$$
H_{1}(Z ; G) \cong \begin{cases}(\mathbb{Z} \otimes G) \oplus \operatorname{Tor}_{1}(\mathbb{Z}, G) \cong G \oplus 0 \cong G & \text { if } \quad \operatorname{dim} V=2 \\ ((\mathbb{Z} / 2 \mathbb{Z}) \otimes G) \oplus \operatorname{Tor}_{1}(\mathbb{Z}, G) \cong G / 2 G & \text { if } \quad \operatorname{dim} V>2\end{cases}
$$

Remark 2.3. Let $\mathcal{H}=\{h, \star, \delta\}$ be a homology theory in the sense of Eilenberg and Steenrod [9, p. 10-12] (see also [13, Section I-2]). Let us to assume that $G$ is the coefficient group of this homology theory and that $\mathcal{H}$ is defined in the admissible category of all the topological pairs, or at least in an admissible category having sufficient topological pairs and maps. It is well known that the one-dimensional homology groups $h_{1}\left(\mathbb{P}^{n}\right)$ of the real projective spaces $\mathbb{P}^{n}$ are given as follows

$$
h_{1}\left(\mathbb{P}^{n}\right) \cong \begin{cases}G & \text { if } n=1 \\ G / 2 G & \text { if } n \neq 1 .\end{cases}
$$

Taking into account Remark 2.2, we see that if $G$ is a given abelian group and $V=\mathbb{R}^{n+1}$ then the singular homology groups $H_{1}(Z ; G)$ and $H_{1}\left(\mathbb{P}^{n} ; G\right)$ are isomorphic. This is not a casual coincidence and it can be derived from the homotopy equivalence of $Z$ and $\mathbb{P}^{n}$. In fact, it is easy to show that, with independence of the possible finite dimensionality of $V$, we always can exhibit a homotopy equivalence of this kind involving the topological space $Z$ given as in Lemma 2.1. In order to prove this, we first recall that if $V$ is a real normed space then the projective space $\mathbb{P}(V)$ is defined as the quotient topological space of the equivalence classes of $V \backslash\{0\}$ relative to the relation $\sim$ defined by $x \sim x^{\prime}$ if and only if $\mathbb{R} x=\mathbb{R} x^{\prime}$. Alternatively, we can define $\mathbb{P}(V)$ as the quotient topological space obtained from the sphere $S$ of $V$ by identifying each pair of antipodal points of $S$. Now we will denote by $p_{Z}: V \backslash\{0\} \rightarrow Z$ and $p_{\mathbb{P}(V)}: V \backslash\{0\} \rightarrow \mathbb{P}(V)$ the corresponding projection maps onto $Z$ and $\mathbb{P}(V)$. Let $j: \mathbb{P}(V) \rightarrow Z$ and $r: Z \rightarrow \mathbb{P}(V)$ be the continuous maps given by $j\left(p_{\mathbb{P}(V)}(x)\right)=p_{Z}(x /\|x\|)$ and $r\left(p_{Z}(x)\right)=p_{\mathbb{P}(V)}(x)$ for all $x$ in $V \backslash\{0\}$. We assert that $j$ and $r$ are inverse homotopy equivalences. Indeed, since $(1-t) x+\frac{t}{\|x\|} x$ is nonzero for all $x \in V \backslash\{0\}$ and $t \in[0,1]$, we can define a continuous map $G:(V \backslash\{0\}) \times[0,1] \rightarrow$ $V \backslash\{0\}$ by

$$
G(x, t)=(1-t) x+\frac{t x}{\|x\|} .
$$

Since $G(-x, t)=-G(x, t)$ for any $(x, t) \in(V \backslash\{0\}) \times[0,1]$, we have a continuous map $H: Z \times[0,1] \rightarrow Z$ such that $H\left(p_{Z}(x), t\right)=p_{Z}(G(x, t))$. Furthermore, $H:$ Id $\simeq$ $j \circ r$. On the other hand, $r \circ j=\mathrm{Id}$. Thus, $Z$ and $\mathbb{P}(V)$ are homotopy equivalent. In particular, $H_{1}(Z ; G) \cong H_{1}(\mathbb{P}(V) ; G)$.

\section{The square map}

Let $A$ be a commutative real or complex nearly absolute valued algebra with all elements having square roots and $q: A \backslash\{0\} \rightarrow A \backslash\{0\}$ the map given by $q(x)=x^{2}$ 
for any $x \in A \backslash\{0\}$. Then the map $q$ is surjective and, in addition, the preimage by $q$ of each element of $A \backslash\{0\}$ is a subset of exactly two elements. Indeed, as a consequence of (1), $A$ has no nonzero zero divisors. So if $x$ and $u$ are nonzero elements of $A$ such that $x^{2}=u^{2}$, then $0=x^{2}-u^{2}=(x+u)(x-u)$. Therefore, $u= \pm x$. In this section we will look more closely at the map $q$. So we will prove that if, in addition, $A$ is complete then $q$ is a closed map, so as the map $g: Z \rightarrow A \backslash\{0\}$ induced by $q$ on the space of orbits $Z$ introduced as in section 2. Furthermore, we show in Corollary 3.3 that the existence of square roots for all the elements of a commutative nearly absolute valued algebra is preserved by completions.

Now let $A$ be a commutative complete nearly absolute valued division algebra. In [5], the proof that the image of the square map $q: A \backslash\{0\} \rightarrow A \backslash\{0\}\left(q: z \mapsto z^{2}\right)$ is a closed subset of $A \backslash\{0\}$, is based in the property that we state in the following proposition for the convenience of reader.

Proposition 3.1 ([5, Proof of Proposition 1.2]). Let A be a commutative complete nearly absolute valued algebra, $y$ a nonzero element of $A$ and $\left\{x_{n}\right\}$ a sequence of $A \backslash\{0\}$ such that $y=\lim _{n \rightarrow \infty} x_{n}^{2}$. Then there exists a convergent sequence $\left\{v_{n}\right\}$ of elements of $A$ with each $v_{n}$ agreeing up to the sign with $x_{n}$.

Another easy consequence of Proposition 3.1 is the lemma below.

Lemma 3.2. Let $A$ be a commutative complete nearly absolute valued algebra and $q: A \backslash\{0\} \rightarrow A \backslash\{0\}$ the map transforming each $x$ in its square $x^{2}$. If ${ }^{-}$denotes the closure operator in the topological subspace $A \backslash\{0\}$ and $T$ is a subset of $A \backslash\{0\}$ such that $-z \in T$ for all $z \in T$, then $\overline{q(T)} \subset q(\bar{T})$.

Proof. Let $y$ be an arbitrary element of $\overline{q(T)}$. So there exists a sequence $\left\{x_{n}\right\}$ of elements of $T$ such that $y=\lim _{n \rightarrow \infty} q\left(x_{n}\right)$. By Proposition 3.1, there exists a convergent sequence $\left\{v_{n}\right\}$ of elements of $T$ such that $y=\lim _{n \rightarrow \infty} v_{n}^{2}$. If $x=\lim _{n \rightarrow \infty} v_{n}$ then $y=x^{2}$ and $x \in \bar{T}$. Therefore, $y \in q(\bar{T})$ and $\overline{q(T)} \subset q(\bar{T})$.

Corollary 3.3. The completion $B$ of a commutative real or complex nearly absolute valued algebra $A$ is a commutative nearly absolute valued algebra, all of whose elements have square roots in the case that this is so for all elements of $A$.

Proof. Obviously $B$ is a commutative nearly absolute valued algebra such that $k\|z\|\|w\| \leqslant\|z w\|$ for all $z, w \in B$ if this is so for each pair of arbitrary elements $z$ and $w$ in $A$. Assume all elements of $A$ have square roots. Let $q: B \backslash\{0\} \rightarrow B \backslash\{0\}$ be the square map of $B \backslash\{0\}$. Viewing $A$ as a subset of $B$, then for the subset $T=A \backslash\{0\}$ we have $\overline{q(T)}=\bar{T}=B \backslash\{0\}$. By Lemma 3.2, $B \backslash\{0\}=q(B \backslash\{0\})$. Thus, all nonzero elements of $B$ have square roots.

Corollary 3.4. Let $A$ be a commutative complete nearly absolute valued algebra, $q: A \backslash\{0\} \rightarrow A \backslash\{0\}$ the map given by $q: x \mapsto x^{2}$, and $Z$ the space of orbits given as in Lemma 2.1. Then both the map $q$ and the map $g: Z \rightarrow A \backslash\{0\}$ induced by $q$ are closed maps.

Proof. We first show the closed character of the map $g$. Let $p: A \backslash\{0\} \rightarrow Z$ be the projection map, $F$ a closed subset of $Z$ and $T=p^{-1}(F)$. By continuity of $p$, the set $T$ is a closed subset of $A \backslash\{0\}$. Furthermore, $-v \in T$ if $v \in T$. Taking into account 
Lemma 3.2, we obtain that the set $g(F)=g(p(T))=q(T)$ is a closed subset of $A \backslash$ $\{0\}$. Thus, $g$ is a closed map. In order to prove that $q$ is also a closed map, let us consider a closed subset $L$ of $A \backslash\{0\}$. We have $p^{-1}(p(L))=L \cup(-L)$, where $-L$ is the subset of the elements $x$ of $A \backslash\{0\}$ such that $-x \in L$. So $p^{-1}(p(L))$ is a closed subset of $A \backslash\{0\}$. In consequence, $p(L)$ is a closed subset of $Z$. So, $q(L)=g(p(L))$ is a closed subset. Therefore, $q$ is a closed map.

Remark 3.5. Lemma 3.2 and Corollary 3.4 can be applied in the case of a commutative finite-dimensional real division algebra $A$. Nevertheless, in this case both results can be obtained more quickly using the compactness of the closed and bounded subsets of $A$, without need to invoke Proposition 3.1. Furthermore, under the requirement of finite-dimensionality the inclusion $\overline{q(T)} \subset q(\bar{T})$ can be established directly without the additional assumption on the set $T$ of $-z \in T$ for all $z \in T$. Indeed, assume $k>0$ is a real number such that (1) holds. Let $\left\{x_{n}\right\}$ and $y$ be as in the proof of Lemma 3.2. Let $\varepsilon>0$ be a real number such that $\varepsilon<\|y\|$ and $N(\varepsilon)$ a positive integer such that $\left|\left\|x_{n}^{2}\right\|-\|y\|\right|<\varepsilon$, for any $n \geqslant N(\varepsilon)$. So

$$
\sqrt{\|y\|-\varepsilon} \leqslant\left\|x_{n}\right\| \leqslant \sqrt{\frac{\left\|x_{n}^{2}\right\|}{k}}<\sqrt{\frac{\varepsilon+\|y\|}{k}}
$$

for all $n \geqslant N(\varepsilon)$. Thus, with the possible exception of a finite number of elements, the sequence $\left\{x_{n}\right\}$ is contained in the compact subset

$$
\left\{v \in A: \quad \sqrt{\|y\|-\varepsilon} \leqslant\|v\| \leqslant \sqrt{\frac{\varepsilon+\|y\|}{k}}\right\} .
$$

In consequence, $\left\{x_{n}\right\}$ has some cluster point $x$. Now $x \in \bar{T}$ and $y=q(x)$, which shows that $\overline{q(T)} \subset q(\bar{T})$. In particular, $q$ is a closed map. From this it can be easily obtained that $g$ is also a closed map. Indeed, let $p_{Z}: A \backslash\{0\} \rightarrow Z$ be the projection map and $L$ a closed subset of $Z$. Then $C=p_{Z}^{-1}(L)$ so as $q(C)$ are closed subsets. In consequence, $g(L)=g\left(p_{Z}(C)\right)=q(C)$ and so this set is closed. Therefore, $g$ is a closed map.

\section{The main theorem}

Next we establish the main result of this paper. Let $A$ be a given commutative nearly absolute valued algebra with all its elements having square roots. In order to prove that $A$ is a two-dimensional real division algebra, we associate a certain algebraic situation (namely, the existence of some isomorphism between the first integer singular homology groups $H_{1}(Z)$ and $\left.H_{1}(A \backslash\{0\})\right)$, which is impossible in the remaining dimensions.

Theorem 4.1. Let $A$ be a commutative nearly absolute valued algebra. Then all elements of $A$ have square roots if and only if $A$ is a two-dimensional real division algebra.

Proof. It is well known that if $A$ is a two-dimensional real commutative division algebra then each element of $A$ has square roots (see [17, Lemma 8.6.4] or [5, Propositions 1.2 and 1.1]).

Assume now that $A$ is a commutative real nearly absolute valued algebra with each element having square roots. Since $\mathbb{R}$ is the unique one-dimensional real nearly 
absolute valued algebra, we have $\operatorname{dim} A \geqslant 2$. By Corollary 3.3, we can assume without loss in generality that $A$ is complete. Let $g: Z \rightarrow A \backslash\{0\}$ be the bijective continuous map induced by the square map $q: A \backslash\{0\} \rightarrow A \backslash\{0\},\left(q: x \mapsto x^{2}\right)$ on the topological space of orbits given as in Lemma 2.1. By Corollary 3.4, $g$ is a closed map. So $g$ is a homeomorphism and, in consequence, the induced homomorphism $g_{\star}: H_{1}(Z) \rightarrow$ $H_{1}(A \backslash\{0\})$ between the first integer singular homology groups is an isomorphism. By Lemma 2.1 this implies $\operatorname{dim} A=2$. Now the fact that $A$ has no nonzero zero divisors implies to be $A$ a division algebra.

Remark 4.2. Neither commutativity nor the existence of square roots of each element can be dropped in Theorem 4.1. See [14, Example 1.1] or [4, Example 2.7.64] for an example of a commutative nearly absolute valued algebra with infinite dimension. On the other hand, the real division algebra $\mathbb{H}$ of quaternions is the most easy example of a non-commutative finite-dimensional nearly absolute valued algebra with all elements having square roots.

Remark 4.3. Theorem 4.1 implies that $\operatorname{dim} A=2$ when $A$ is a commutative finite-dimensional real division algebra not isomorphic to $\mathbb{R}$. Nevertheless, in this case it is possible to give a more direct proof. Indeed, if $\mathbb{P}$ is the projective space of $A$ and $p_{\mathbb{P}}: A \backslash\{0\} \rightarrow \mathbb{P}$ its corresponding projection map, then the map $\tilde{g}: \mathbb{P} \rightarrow S$ defined by $\tilde{g}: p_{\mathbb{P}}(x) \mapsto x^{2} /\left\|x^{2}\right\|$ is a bijective continuous map. By compactness of $\mathbb{P}, \tilde{g}$ is a homeomorphism. Thus, $\tilde{g}_{\star}$ is an isomorphism. We observe that, under the above assumptions, this allows us to obtain that $A$ is a two-dimensional division algebra. Indeed, it is well known that the first integer singular homology groups of $\mathbb{P}$ and $S$ are isomorphic only in the case that $\operatorname{dim} A=2$ (see Lemma 2.1 and Remark 2.3; or [13, p. 137], [2, p. 179]).

It can be seen that the above argument has certain similarities with that of the proof of Theorem 4.1. In both cases, that the dimension of $A$ is 2 is a consequence of the existence of a certain isomorphism between integer singular 1-dimensional homology groups. In one case this isomorphism is $\tilde{g}_{\star}$, in the other it is $g_{\star}$. An accurate analysis shows that this should not surprise us, since as a consequence of the following statement $(\star), \tilde{g}_{\star}$ is an isomorphism if and only $g_{\star}$ is.

(*) Let $A$ be a commutative real normed algebra, with unit sphere $S$ and projective space $\mathbb{P}$, whose space of orbits introduced as in section 2 is denoted by $Z$. Assume $A$ has no nonzero elements with zero square. Let $p_{Z}: A \backslash\{0\} \rightarrow Z$ and $p_{\mathbb{P}}: A \backslash\{0\} \rightarrow \mathbb{P}$ be the corresponding projection maps, and $r: Z \rightarrow \mathbb{P}$ and $\tilde{r}: A \backslash\{0\} \rightarrow S$ the maps defined by

$$
r: p_{Z}(x) \mapsto p_{\mathbb{P}}(x), \quad \tilde{r}: x \mapsto \frac{x}{\|x\|}
$$

for all $x \in A \backslash\{0\}$. Let $g: Z \rightarrow A \backslash\{0\}$ be the map induced by the square map $q: A \backslash\{0\} \rightarrow A \backslash\{0\}\left(q: x \mapsto x^{2}\right)$ and $\tilde{g}: \mathbb{P} \rightarrow S$ the map defined by $\tilde{g}: p_{\mathbb{P}}(x) \mapsto x^{2} /\left\|x^{2}\right\|$. Then $g_{\star}: H_{1}(Z) \rightarrow H_{1}(A \backslash\{0\})$ is an isomorphism if and only if $\tilde{g}_{\star}: H_{1}(\mathbb{P}) \rightarrow H_{1}(S)$ is.

Indeed, it is easy to check the commutativity of the square

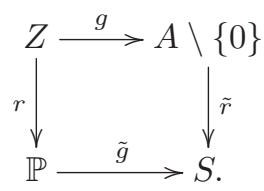


This implies that the following diagram of homomorphisms between integer singular homology groups

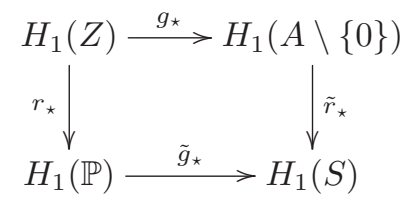

is also commutative. Since $r$ and $\tilde{r}$ are homotopy equivalences, the homomorphisms $r_{\star}$ and $\tilde{r}_{\star}$ are both isomorphisms. In consequence, $g_{\star}$ is an isomorphism if and only if $\tilde{g}_{\star}$ is, which proves $(\star)$.

Corollary 4.4. Let A be a commutative complex nearly absolute valued algebra. Then each element of $A$ has square roots if and only if $A$ is isometrically isomorphic to $\mathbb{C}$.

Proof. Obviously $\mathbb{C}$ is a commutative complex nearly absolute valued algebra where each element has some square root. Now let $A$ be a commutative complex nearly absolute valued algebra with all elements having square roots. By Theorem 4.1, the dimension of $A$ viewed as a real algebra is 2 . In consequence, $A$ is isometrically isomorphic to $\mathbb{C}$.

Corollary 4.4 allows us to focus exclusively on the real case. Now we give some consequences of Theorem 4.1 in this context.

Corollary 4.5. If $A$ is a commutative real algebra then the following assertions are equivalent:

1. A is a division nearly absolute valued algebra.

2. Either $A$ is isomorphic to $\mathbb{R}$ or $A$ is a nearly absolute valued algebra with all elements having some square root.

3. $A$ is a division algebra and $\operatorname{dim} A \leqslant 2$.

4. A is a finite-dimensional division algebra.

5. A is a nearly absolute valued algebra with some element a such that $L_{a}$ has dense image.

Proof. The equivalence $1 \Leftrightarrow 5$ was established in [5, Corollary 2.6]. $3 \Rightarrow 4$ is obvious. The implication $4 \Rightarrow 1$ is a consequence of the compact character of the spheres of the finitedimensional normed vector spaces. $2 \Rightarrow 1$ and $2 \Rightarrow 3$ are consequences of Theorem 4.1 . Finally, $1 \Rightarrow 2$ is a consequence of the fact that if $\operatorname{dim} A \geqslant 2$ then the square map $q: A \backslash\{0\} \rightarrow A \backslash\{0\}$ is a surjective map (see Proposition 1.1).

Remark 4.6. Hopf's Theorem [12] asserting that each commutative finite-dimensional real division algebra has dimension $\leqslant 2$ is implication $4 \Rightarrow 3$ in Corollary 4.5.

Since, up to isomorphism, $\mathbb{C}$ and $\stackrel{\star}{\mathbb{C}}$ are the unique commutative real normed division algebras of dimension 2 whose norm satisfies $\left\|x^{2}\right\|=\|x\|^{2}$ for each $x$ ([5, Proposition 3.1]), we obtain the corollary below.

Corollary 4.7. Let $A$ be a commutative real nearly absolute valued algebra whose elements have square roots. If $\left\|x^{2}\right\|=\|x\|^{2}$ for all $x \in A$, then $A$ is isomorphic to $\mathbb{C}$ or $\stackrel{\star}{\mathbb{C}}$. 
Finally, taking into account [16, Theorem 3.7] we obtain the next corollary.

Corollary 4.8. Let $A$ be a commutative real algebra with no nonzero zero divisors. If the subalgebra generated by each nonzero element of $A$ is a nearly absolute valued algebra with all elements having square roots, then $\operatorname{dim} A=2$.

\section{Acknowledgments}

The authors wish to acknowledge to professors Miguel Cabrera García and Ángel Rodríguez Palacios for the carefully reading of the manuscript and their valuable comments. They also are very grateful to the referee for his helpful suggestions. All have contributed to the improvement of this paper.

During the realization of this work the first author was partially supported by the Ministerio de Economía y Competitividad (MTM2013-45588-C3-P and MTM201783506-C2-2-P).

During the realization of this work the second author was partially supported by the Ministerio de Economía y Competitividad (MTM2017-83506-C2-2-P).

\section{References}

[1] S.C. Althoen and L.D. Kugler. When is $\mathbb{R}^{2}$ a division algebra? Amer. Math. Monthly, 90:625-635, 1983.

[2] E. Artin and H. Braun. Leçons de Topologie Algébrique. Les Presses de l'Université du Québec, 1973.

[3] G. Benkart, D.J. Britten, and M.J. Osborn. Real flexible division algebras. Canad. J. Math., XXXIV(3):550-588, 1982.

[4] M. Cabrera and A. Rodríguez. Non-associative normed algebras: Volume 1 the Vidav-Palmer and Gelfand-Naimark theorems, volume 154 of Encyclopedia Math. Appl. Cambridge University Press, 2014.

[5] J.A. Cuenca and F. Miguel. On a theorem by Hopf. Linear Algebra Appl. (2018). https://doi.org/10.1016/j.laa.2018.04.008.

[6] E. Darpö. On the classification of real flexible division algebras. Colloq. Math., 105(1):1-17, 2006.

[7] E. Darpö and E. Dieterich. Real commutative division algebras. Algebr. Represent. Theory, 10:179-196, 2007.

[8] H.-D. Ebbinghaus, H. Hermes, F. Hirzebruch, M. Koecher, K. Mainzer, J. Neukirch, A. Prestel, and R. Remmert. Numbers, volume 123 of Grad. Texts in Math. Springer-Verlag, 1991.

[9] S. Eilenberg and N. Steenrod. Foundations of algebraic topology. Princenton Univ. Press, 1952.

[10] A. Hatcher. Algebraic Topology. Electronic version in https://www.math . cornell. edu/ hatcher/AT/AT.pdf.

[11] M. Hübner and H.P. Petersson. Two-dimensional real division algebras revisited. Beitr. Algebra Geom., 45:29-36, 2004. 
[12] H. Hopf. Ein topologischer Beitrag zur reellen algebra. Comment. Math. Helv., 13:219-238, 1940.

[13] Sze-Tsen Hu. Homology Theory: A first course in algebraic topology. Holden Day, 1966.

[14] A. Kaidi, M. Ramírez, and A. Rodríguez. Nearly absolute-valued algebras. Comm. Algebra, 30(7):3267-3284, 2002.

[15] I.L. Kantor and A.S. Solodovnikov. Hypercomplex numbers. An elementary introduction to algebras. Springer-Verlag, 1989.

[16] A. Moutassim and M. Benslimane. Generalization of the Hopf commutative theorem. Comm. Algebra, 45(2):883-888, 2017.

[17] J. Roe. Winding around. The winding number in topology, geometry, and analysis, volume 76 of Stud. Math. Libr. American Mathematical Society, 2015.

[18] R.M. Switzer. Algebraic Topology. Springer-Verlag, Berlin Heidelberg New York, 1975.

José Antonio Cuenca Mira cuenra@agt.cie.uma.es

Departamento de Álgebra, Geometría y Topología, Facultad de Ciencias, Universidad de Málaga, Málaga, 29071, Spain

Francisca Miguel García fcamiguel@uma.es

Departamento de Economía Aplicada (Matemáticas), Facultad de Ciencias Económicas, Universidad de Málaga, Málaga, 29013, Spain 